\title{
Double-Ring Marker Based 3D Pose Estimation For Rod-Shaped MIS Instrument From a Single 2D Image
}

\author{
Ming-Juan Yu ${ }^{\mathrm{a}}$, Jia-Cyuan Syu ${ }^{\mathrm{a}}$,Guo-Shing Lin ${ }^{\mathrm{b}}$, Wen-Nun Lie ${ }^{\mathrm{c}}$, Jen-Chun Huang ${ }^{\mathrm{c}}$, Huan-Ting Chang ${ }^{\mathrm{a}}$, Jack Liu ${ }^{\mathrm{d}}$, \\ Chuen-Horng Lin ${ }^{\mathrm{e}}$, Day-Fann Shen ${ }^{\mathrm{a}}$ \\ ${ }^{a}$ Department of Electrical Engineering, National Yunlin University of Science and Technology, Douliu,640, Taiwan \\ ${ }^{\mathrm{b}}$ Department of Information Engineering, Da-Yeh University, Dacun,515, Taiwan \\ ${ }^{c}$ Department of Electrical Engineering, National Chung Cheng University,Tamio,621 , Taiwan \\ ${ }^{\mathrm{d}}$ Show Chwan Hospital Incubation Center, Lukang,505, Taiwan \\ ${ }^{\mathrm{e}}$ Department of Computer Science and information Engineering, National Taichung University of Science and \\ Technology, Taichung City, 404, Taiwan
}

*Corresponding Author: shendf@yuntech.edu.tw

\begin{abstract}
In this paper, we propose a hologram like Double-Ring Marker based method which can estimate the six (6) 3D pose parameters of a Rod-shaped body such as MIS (Minimally Invasive Surgery) instrument using just a single 2D image. The six 3D pose parameters are derived from a set of geometric equations that the predefined Double-Ring Markers on the rod be projected onto the image plan through the camera's perspective transformation. Compared to existing MIS pose estimation methods, which normally require time consuming processes including depth estimation, instrument identification and position parameters estimation as the pre-processing, the proposed Double-Rings marker based algorithm is very efficient and accurate.
\end{abstract}

Keywords: Double-Ring-Markers, Rod-Shaped object, Minimally invasive surgery (MIS), depth information, 3D Position parameters estimation, stereo imaging.

\section{Introduction}

Minimally Invasive Surgery (MIS) become very popular because it can greatly reduce the pain and recovery span for patients. 3D pose estimation and tracking of the MIS instrument is an important technique toward remote surgery through robotics [1-5].

Among the 3D pose parameters, the depth information of MIS instrument is currently obtained through the widely adopted stereo-imaging algorithm which requires the stereo cameras system and the time-consuming matching process between left and right (stereo) images of a scene as the pre-process. In this paper, we proposed a Double-Ring-Markers based method for estimation of 3D pose parameters for any rod-shaped body including the MIS instrument. The proposed Double-Ring-Markers system requires only a single $2 \mathrm{D}$ image obtained from a camera, with additional two (double) ring markers of same width but different color painted at a predetermined distance on the surface of the rod-shaped body. Using the predefined information of the two rings and the perspective transformation of the camera, we are able to derive a set of deterministic equations for calculating the six 3D pose parameters of a rod-shaped body. Compared to the stereo camera system which is more costly in hardware and computation, the proposed method can estimate the 3D pose of any rod-shaped body very precisely and very efficiently in terms of both hardware and computational cost.

This paper is organized as follows: In section 2, we describe the system and derive the deterministic equations for calculating the 6 pose parameters. In section 3, we describe the process of the proposed algorithm. In section 4 , we conduct experiments to prove the correctness of the proposed algorithm. Conclusion is made in section 5.

\section{Derivation Of 3D Pose Parameters Of a Rod-Shaped Body}

We assume that the origin of the 3D world coordinate system $(\mathrm{X}, \mathrm{Y}, \mathrm{Z})$ and the 2D image plan coordinate system $(\mathrm{x}, \mathrm{y})$ are coincident as shown in Figure 1(a), where $\mathrm{X}$ and $\mathrm{x}$ and are horizontal axis, $\mathrm{Y}$ and $\mathrm{y}$ are vertical axis, while $\mathrm{Z}$ is the depth axis.

Ring-A and Ring-B (Double-Rings) of the same width $\mathrm{L}$ are painted or marked surrounding the surface near the top (front end) of the Rod-Shaped body at a predefined distance $L_{A B}$ as shown in Figure 1(a)(b). Two different colors easily distinguishable from the scene are assigned to Ring-A and Ring-B respectively for easy and precise 
segmentation of Ring-A and Ring-B in the 2D image.

The 3D pose of a rod-shaped object can be define by six (6) parameters i.e. $\mathrm{X}_{\mathrm{AA} 1}, \mathrm{Y}_{\mathrm{AA} 1}, \mathrm{Z}_{\mathrm{AA} 1}$ and $\alpha, \beta, \gamma$, where $\mathrm{X}_{\mathrm{AA} 1}, \mathrm{Y}_{\mathrm{AA} 1}, \mathrm{Z}_{\mathrm{AA} 1}$ are the $3 \mathrm{D}$ coordinate of the chosen reference point AA1 as shown in Figure 1(b). Points AA1 (and $\mathrm{BB}_{1}$ ) is the intersection of the rod's axis and the plane containing the upper edges of Ring-A (and upper edges of Ring-B). The rod's position and direction is presented by the directional line $\overrightarrow{B B_{1} A A_{1}}$ which pass through the rod's axis.

Since the reference point AA1 is on the rod's axis, it cannot be seen and cannot be detected from the 2D image. Instead, the upper edge point $\mathrm{A} 1$ on the surface of Ring $\mathrm{A}$ with coordinate $\left(\mathrm{X}_{\mathrm{A} 1}, \mathrm{Y}_{\mathrm{A} 1}, \mathrm{Z}_{\mathrm{A} 1}\right)$ as shown in Fig.1(b) is estimated before estimation of the reference $\left(\mathrm{X}_{\mathrm{AA} 1}, \mathrm{Y}_{\mathrm{AAl}}\right.$, $\mathrm{Z}_{\mathrm{AA} 1}$ ). Fortunately, the $3 \mathrm{D}$ point $\mathrm{A} 1$ can be detected in the $2 \mathrm{D}$ image as an intermediate step toward the estimation of the reference point AA1.

Let line $\overrightarrow{B A}$ be the intersection of the rod's surface and the trajectories of the axis line $\overrightarrow{B B_{1} A A_{1}}$ projecting on to the image plan, as shown in Fig. 1(b). Since we assume that the diameters of the rod is even, line $\overrightarrow{B A}$ on the rod's surface is parallel to the axis $\overrightarrow{B B_{1} A A_{1}}$. It is noted that point $\mathrm{A} 1$ is at the intersection of $\overrightarrow{B A}$ and upper edges of Ring A, see Fig. 1(b).

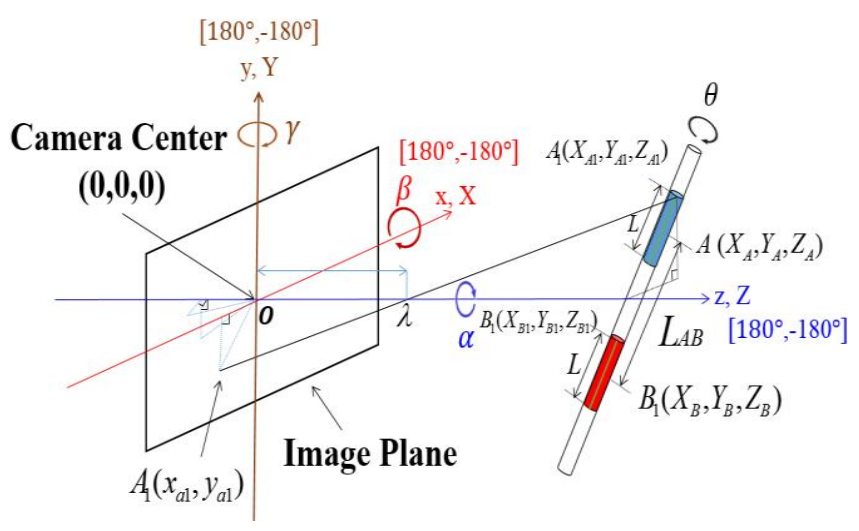

(a)

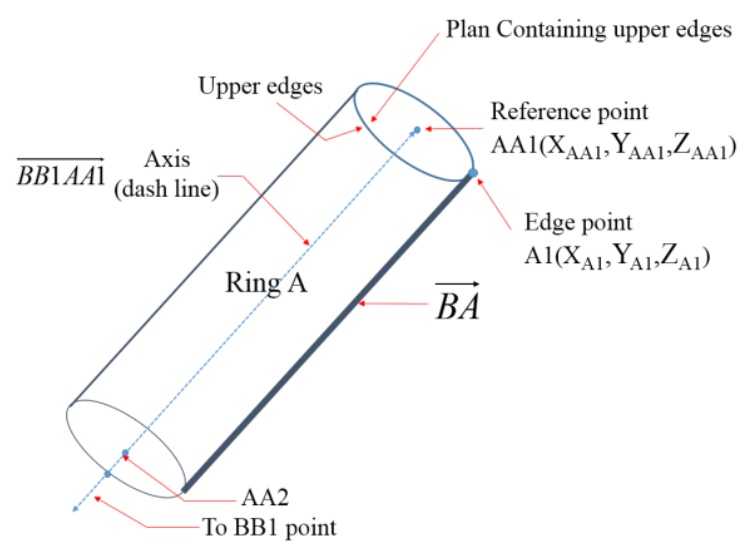

(b)

Fig. 1: (a) The Imaging Relationship of a Rod-Shaped Body with Double-Ring-Markers (b) Reference point AA1 at the intersection of rod's axis and the plan containing the upper edges of Ring-A

Three angles parameters $\alpha, \beta, \gamma$ in degrees as shown in Figure 1(a) are defined in details below: (1) Angle $\alpha$ is the angle between $\mathrm{X}(\mathrm{x})$-axis and the projection of directional line vector $\overrightarrow{\boldsymbol{B A}}$ onto $\mathrm{x}-\mathrm{y}(\mathrm{X}-\mathrm{Y})$ image plan. Angle $\alpha$, rotating around $\mathrm{Z}$ axis, is also referred to as the In-Plan angle. (2) Angle $\beta$ is the angle between $X(x)$-axis and the projection of directional line $\overrightarrow{B A}$ onto $\mathrm{X}(\mathrm{x})-\mathrm{Z}$ plan. Angle $\beta$, rotating around $\mathrm{Y}(\mathrm{y})$-axis, is also referred to as the out-plan angle. (3)Angle $\gamma$ is the angle between $\mathrm{Y}(\mathrm{x})$-axis and the projection of directional line $\overrightarrow{B A}$ onto $\mathrm{Z}-\mathrm{Y}(\mathrm{y})$ plan, rotating around $\mathrm{X}(\mathrm{x})$ axis, also referred to as Out-Plan angle.

These 6 parameters $\left(\mathrm{X}_{\mathrm{AA} 1}, \mathrm{Y}_{\mathrm{AA} 1}, \mathrm{Z}_{\mathrm{AA} 1}\right.$ and $\left.\alpha, \beta, \gamma\right)$ uniquely determine the $3 \mathrm{D}$ position of the Double-Ring marked Rod-Shaped body.

\subsection{Estimation of in-plane angle $\alpha$}

Let the 2D directional line vector $\overrightarrow{b a}$ be the projection of directional 3D line vector $\overrightarrow{B A}$ onto $\mathrm{x}-\mathrm{y}$ image plan, and $\alpha$ is the angle between $\overrightarrow{b a}$ and positive x-axis. $\overrightarrow{b a}$ can be found by connecting image point $\mathrm{A}\left(\mathrm{x}_{\mathrm{a}}, \mathrm{y}_{\mathrm{a}}\right)$ and $\mathrm{B}\left(\mathrm{x}_{\mathrm{b}}, \mathrm{y}_{\mathrm{b}}\right)$, where $\mathrm{A}\left(\mathrm{x}_{\mathrm{a}}, \mathrm{y}_{\mathrm{a}}\right)$ is the centroid of the area that Ring- $\mathrm{A}$ projected onto the image plan through the camera lens of focal length $\lambda$. Similarly, $B\left(x_{b}, y_{b}\right)$ be the centroid of the area that Ring-B projected onto the image plan through the same camera. There are four possibilities of angle $\alpha$ accordingly to the direction of the rod as shown in Fig.2, where $\alpha=\tan ^{-1}\left(\left(\left|y_{a}-y_{b}\right|\right) /\left(\left|x_{a}-x_{b}\right|\right)\right)$

In this paper, we limit our discussion to rod direction $\overrightarrow{B A}$ pointing from left to right and away from the camera, due to page space constraint. 
In this case, $\alpha_{\mathrm{I}}=\tan ^{-1}\left(\left(y_{a}-y_{b}\right) /\left(x_{a}-x_{b}\right)\right)$

(1)

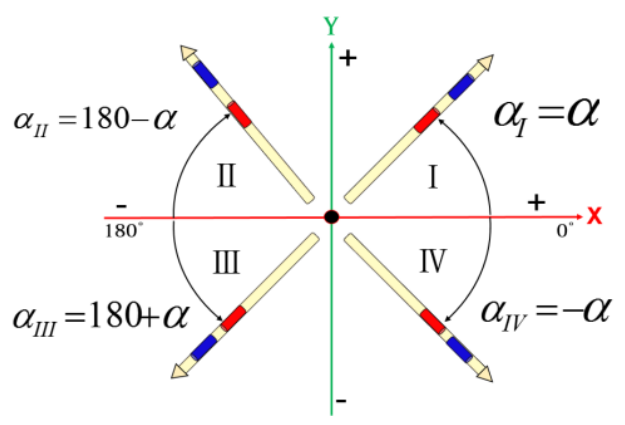

Fig. 2 Four possibilities of pose angle accordingly to direction of the rod

\subsection{Estimation of out-plane angle $\beta$ and depth $\mathrm{Z}_{\mathrm{A} 1}$}

Figure 3 shows the rod pose projected onto the $\mathrm{XZ}$ plane, where $\mathrm{x}_{\mathrm{a} 1}, \mathrm{x}_{\mathrm{a} 2}$ are the $\mathrm{x}$-coordinates of the projected upper and lower edges of Ring-A respectively, while $\mathrm{x}_{\mathrm{b} 1}, \mathrm{x}_{\mathrm{b} 2}$ are that of Ring-B.

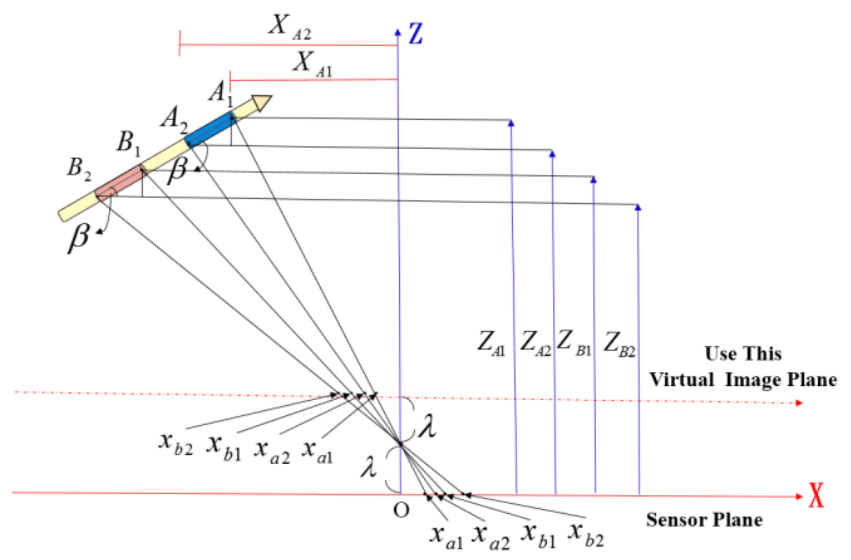

Fig. 3 Projection of 3D rod-shaped body onto x-axis in image plan

Given $\mathrm{x}_{\mathrm{a} 1}, \mathrm{x}_{\mathrm{a} 2}, \mathrm{x}_{\mathrm{b} 1}, \mathrm{x}_{\mathrm{b} 2}$ and camera focal length $\lambda$, width of Ring-A and Ring-B $L$ and length between Ring-A and Ring- $B L_{A B}$, we would like to find the out-plane angle $\beta$ and depth $\mathrm{Z}_{\mathrm{A} 1}$ by using the perspective geometric transformation of the camera.

From Ring A of Figure 3, we have the following relations in $3 \mathrm{D}$ coordinates,

$X_{A 1}-X_{A 2}=L \cos \beta$

Using image perspective geometric relationships, we have

$X_{A 2}=\frac{Z_{A 2}-\lambda}{\lambda} x_{a 2}$

$X_{A 1}=\frac{Z_{A 1}-\lambda}{\lambda} x_{a 1}$

Substitute Eq. (2) (3) to Eq.(1), we get
$\frac{Z_{A 1}-\lambda}{\lambda} x_{a 1}-\frac{Z_{A 2}-\lambda}{\lambda} x_{a 2}=L \cos \beta$

Simplify Eq.(4) further by using the 3D geometric

relationship $Z_{A 2}=Z_{A 1}-L \sin \beta$

After lengthy derivations, we eventually obtain the out-plane angle $\beta$ in Eq.(5) and depth $\mathrm{Z}_{\mathrm{Al}}$ in Eq.(6)

$\beta=\cos ^{-1} \sqrt{\frac{1}{1+F_{I}^{2}}}$

where

$$
\mathrm{F}=\frac{L\left[\frac{1}{\left(x_{a 1}-x_{a 2}\right)}-\frac{1}{\left(x_{b 1}-x_{b 2}\right)}\right] \lambda}{L_{A B}+L\left[\frac{x_{a 2}}{\left(x_{a 1}-x_{a 2}\right)}-\frac{x_{b 2}}{\left(x_{b 1}-x_{b 2}\right)}\right]}
$$

$Z_{A 1}=\left[\lambda\left(L \cos \beta+\left(x_{a 1}-x_{a 2}\right)\right)-L x_{a 2} \sin \beta\right] /\left(x_{a 1}-x_{a 2}\right)$

2.3 Estimation of out-plane angle $\gamma$ and depth $\mathrm{Z}_{\mathrm{A} 1}$

Similarly, given $\mathrm{y}_{\mathrm{a} 1}, \mathrm{y}_{\mathrm{a} 2}, \mathrm{y}_{\mathrm{b} 1}, \mathrm{y}_{\mathrm{b} 2}, \lambda, L$ and $\mathrm{L}_{\mathrm{AB}}$, by using the perspective geometric transformation of the camera on the YZ plane, we obtain the out-plane angle $\gamma$ in Eq.(7) and depth $\mathrm{Z}_{\mathrm{A} 1}$ in Eq.(8) as follows:

$\gamma=\cos ^{-1} \sqrt{\frac{1}{1+G^{2}}}$

Where

$$
\mathrm{G}=\frac{L\left[\frac{1}{\left(y_{a 1}-y_{a 2}\right)}-\frac{1}{\left(y_{b 1}-y_{b 2}\right)}\right] \lambda}{L_{A B}+L\left[\frac{y_{a 2}}{\left(y_{a 1}-y_{a 2}\right)}-\frac{y_{b 2}}{\left(y_{b 1}-y_{b 2}\right)}\right]}
$$

And the depth of point A1

$Z_{A 1}=\left[\lambda\left(\mathrm{L} \cos \gamma+\left(y_{a 1}-y_{a 2}\right)\right)-\mathrm{L} y_{a 2} \sin \gamma\right] /\left(y_{a 1}-y_{a 2}\right)$

Note that $\mathrm{Z}_{\mathrm{A} 1}$ can be obtained either using Eq. (6) or Eq. (8), if $\left|x_{a_{1}}-x_{a 2}\right|>\left|y_{a 1}-y_{a 2}\right|$ then Eq.(6) is used; otherwise, Eq. (8) is used.

\subsection{Estimation of 3D coordinate of reference point AA1}

In Fig.1(b), note that the estimation of point A1's coordinate $\left(\mathrm{X}_{\mathrm{A} 1}, \mathrm{Y}_{\mathrm{A} 1}, \mathrm{Z}_{\mathrm{A} 1}\right)$ is an intermediate step to find the final uniquely defined reference point AA1's coordinate $\left(\mathrm{X}_{\mathrm{AA} 1}, \mathrm{Y}_{\mathrm{AA} 1,} \mathrm{Z}_{\mathrm{AA} 1}\right)$.

Given image coordinate $\left(\mathrm{x}_{\mathrm{a} 1}, \mathrm{y}_{\mathrm{a} 1}\right), \mathrm{Z}_{\mathrm{A} 1}$ and $\lambda$, we would estimate the $3 \mathrm{D}$ coordinates of point $\mathrm{A} 1$ then the reference point AA1 as follows:

Step 1: Estimation of 3D coordinates for $\mathrm{A} 1\left(\mathrm{X}_{\mathrm{A} 1}, \mathrm{Y}_{\mathrm{A} 1}, \mathrm{Z}_{\mathrm{A} 1}\right)$ 
on rod's surface. Since $Z_{\mathrm{A} 1}$, the depth of point $A 1$, is available by eq.(6) or eq.(8) (see sec. 2.3), we may obtain $\mathrm{X}_{\mathrm{A} 1}$ and $\mathrm{Y}_{\mathrm{A} 1}$ using the inverse perspective transformation,

$$
\begin{aligned}
& X_{A 1}=\frac{x_{a 1}\left(Z_{A 1}-\lambda\right)}{\lambda} \\
& Y_{A 1}=\frac{y_{a 1}\left(Z_{A 1}-\lambda\right)}{\lambda}
\end{aligned}
$$

Step 2: Estimation of 3D coordinates for the reference point

$$
\begin{aligned}
A A_{1}\left(X_{A A 1}, Y_{A A 1}, Z_{A A 1}\right) \cdot \\
\mathrm{X}_{\mathrm{AA} 1}=\mathrm{X}_{\mathrm{A} 1}+\Delta \mathrm{x} \\
\mathrm{Y}_{\mathrm{AA} 1}=\mathrm{Y}_{\mathrm{A} 1}+\Delta \mathrm{y} \\
\mathrm{Z}_{\mathrm{AA} 1}=\mathrm{Z}_{\mathrm{A} 1}+\Delta \mathrm{Z}
\end{aligned}
$$

If $\mathrm{x}_{\mathrm{a} 1}>=0$ (reference point at right side of focal point) then $\Delta x=R \sin \beta$ else $\Delta x=-R \sin \beta$; If $\mathrm{y}_{\mathrm{a} 1}>=0$ (reference point at upper side of focal point) then $\Delta y=R \sin \gamma$ else $\Delta y=-R \sin \gamma$, if $\left|\mathrm{x}_{\mathrm{a} 1}{ }^{-} \mathrm{x}_{\mathrm{a} 2}\right|>\left|\mathrm{y}_{\mathrm{a} 1}-\mathrm{y}_{\mathrm{a} 2}\right|$ (the larger the difference the better the precision) then $\Delta z=R \cos \beta$ else $\Delta z=R \cos \gamma$ and $\mathrm{R}$ is the radius of the rod.

Thus, we have obtained the six (6) 3D pose parameters of the Double-Ring Marked rod-shaped object: $\mathrm{X}_{\mathrm{AA} 1}, \mathrm{Y}_{\mathrm{AA} 1}$, $\mathrm{Z}_{\mathrm{AA} 1}$ and $\alpha, \beta, \gamma$.

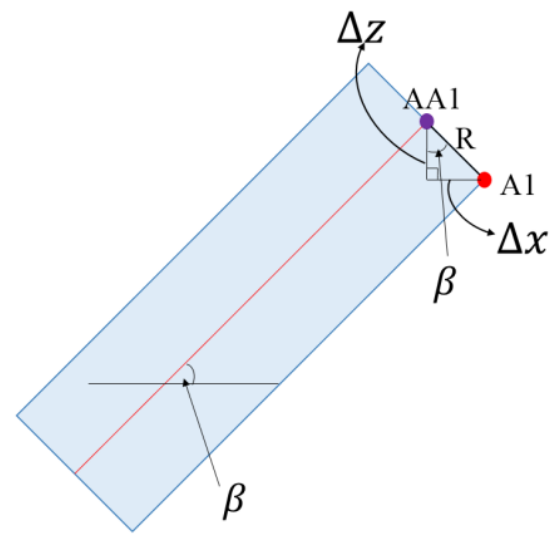

Fig. 3. The geometric relationship between point A1 and the reference point AA1 From Figure 3 ,

\section{The Algorithm}

Given the system described in section 2, the $3 \mathrm{D}$ pose described by the 6 parameters namely $\left(\mathrm{X}_{\mathrm{AA} 1}, \mathrm{Y}_{\mathrm{AA} 1}, \mathrm{Z}_{\mathrm{AA} 1}\right.$, $\alpha, \beta, \gamma)$ of a Double-Ring marked rod can be estimated using just a single 2D image $\mathrm{f}(\mathrm{x}, \mathrm{y})$ as follows:

Step1: (a) Capture the double ring marked rod by a camera, then input the image or a video frame $f(i, j)$ of size $M, N$ and (b) Convert $f(j, i)$ to $f(x, y)$ which has coordinate origin the center of the image i.e.
$\mathrm{x}=\mathrm{j}-\mathrm{M} / 2$ and $\mathrm{y}=\mathrm{N} / 2-\mathrm{i}$. shown in Figure 4.

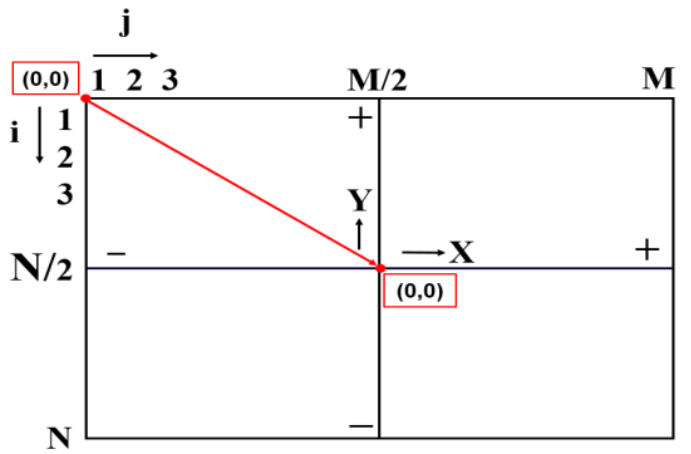

Fig4. Convert coordinates

Step2. (a) Segment the areas of Ring A and Ring B of the rod separately and compute the centroids of Ring A and Ring B accordingly. (b) obtain the projected 2D line $\overrightarrow{b a}$ by connecting the two centroids. (c) Find the edges of Ring A and Ring B.

Step3: Obtain the intersections of line $\overrightarrow{\boldsymbol{b} \boldsymbol{a}}$ with Ring A front edge $\mathrm{A} 1\left(\mathrm{x}_{\mathrm{a} 1}, \mathrm{y}_{\mathrm{a} 1}\right)$ and rear edge $\mathrm{A} 2\left(\mathrm{x}_{\mathrm{a} 2}, \mathrm{y}_{\mathrm{a} 2}\right)$ as well as the intersections of line $\overrightarrow{b a}$ with Ring $B$ front edge $\mathrm{B} 1\left(\mathrm{x}_{\mathrm{b} 1}, \mathrm{y}_{\mathrm{b} 1}\right)$ and rear edge $\mathrm{B} 2\left(\mathrm{x}_{\mathrm{b} 2}, \mathrm{y}_{\mathrm{b} 2}\right)$ as shown in Figure 5.

Step 4: Distance metric conversion. Convert coordinates

$\mathrm{x}_{\mathrm{a} 1}, \mathrm{y}_{\mathrm{a} 1}, \mathrm{X}_{\mathrm{a} 2}, \mathrm{y}_{\mathrm{a} 2}, \mathrm{X}_{\mathrm{b} 1}, \mathrm{y}_{\mathrm{b} 1}, \mathrm{x}_{\mathrm{b} 2}, \mathrm{y}_{\mathrm{b} 2}$ in pixels to $\mathrm{mm}$ $(10 \mathrm{~mm}=1 \mathrm{~cm})$ using the image resolution and camera sensor size information. For example, an image with resolution 960 x 720 and camera sensor size: $4.5 \mathrm{~mm} * 3.4 \mathrm{~mm}$, then the conversion factors $\mathrm{dy}=4.5 / 960 \mathrm{~mm} /$ pixel and $\mathrm{dx}=3.4 / 720 \mathrm{~mm} / \mathrm{pixel}$ respectively.

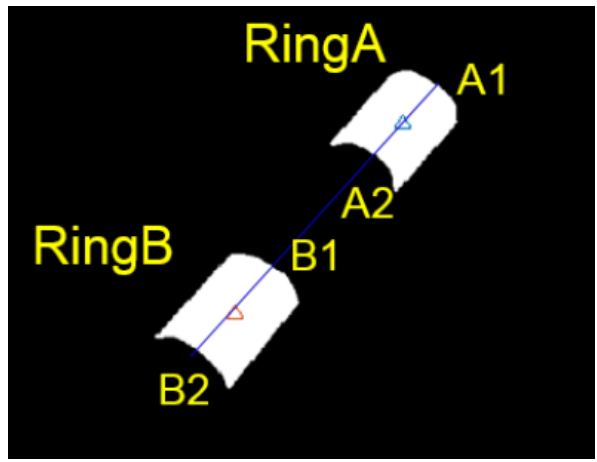

Fig. 5. Intersections of line $\overrightarrow{b a}$ and front and rear edges of Ring A (A1\&A2 respectively) and Ring B (B1\&B2).

Step5: Estimate ange $\alpha$ using Eq.(1) in sec. 2.1

Step6: Estimate ange $\beta$ using Eq.(24) in sec. 2.2

Step7: Estimate angle $\gamma$ using Eq. (27) in sec. 2.3

Step8: Estimate $\mathrm{Z}_{\mathrm{A} 1}$. if $\left|\mathrm{x}_{\mathrm{a} 1}-\mathrm{x}_{\mathrm{a} 2}\right|>\left|\mathrm{y}_{\mathrm{a} 1}-\mathrm{y}_{\mathrm{a} 2}\right|$ then Eq.(6) is used; otherwise, Eq. (8) is used. 
Step9: Estimation of 3D coordinates of reference point $\mathrm{AA} 1\left(\mathrm{X}_{\mathrm{AA} 1}, \quad \mathrm{Y}_{\mathrm{AA} 1}, \quad \mathrm{Z}_{\mathrm{AA} 1}\right)$ using eq.(9)(10)(11) as described in sec. 2.4 .

The six (6) 3D pose parameters namely $\left(\mathrm{X}_{\mathrm{AA} 1}, \mathrm{Y}_{\mathrm{AA} 1}, \mathrm{Z}_{\mathrm{AA} 1}\right.$, $\alpha, \beta, \gamma)$ of a Double-Ring marked rod can be estimated with a single 2D image as described above. In addition, the tracking of the rod position can be achieved by repeating the above algorithm to each video frame.

\section{Experiments}

\subsection{Experimental Set ups}

The purpose of the experiment is to prove the correctness of the derived equations and evaluation of the proposed algorithm. Figure 5 shows the experimental setup as well as the tools for measurements of the six 3D pose parameters.

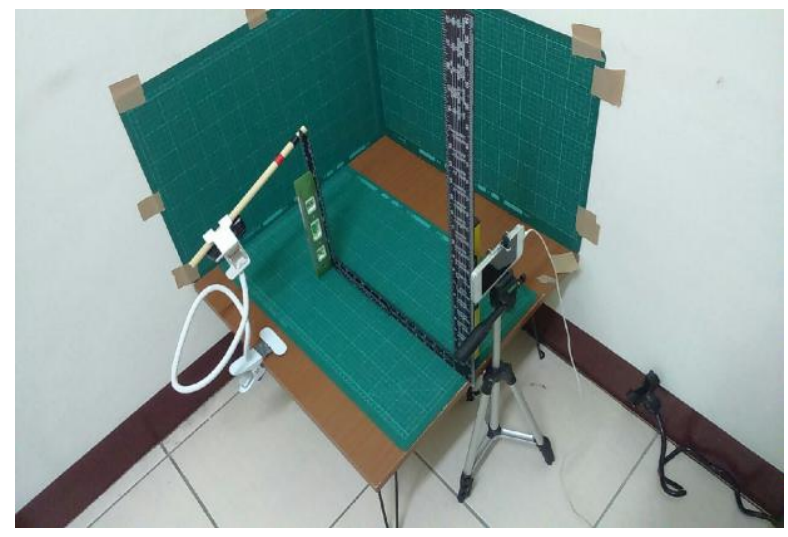

Fig. 5. Experimental Setups

The Apple iPhone 4S camera is used in the experiments. With focal length $\lambda$ of $4.3 \mathrm{~mm}$, width/height ratio: $4: 3$; resolution: $960 \mathrm{x} 720$, sensor size: $4.5 \mathrm{~mm} * 3.4 \mathrm{~mm}$.

\subsection{Experimental Results}

While the 6 estimated parameters are obtained by the proposed algorithm which are unique for a given pose, the measured parameters are the average parameters measured by $\mathrm{N}$ observers ( $\mathrm{N}=2$ in this experiment). A common set of observation procedure is required to reduce the discrepencies among the measured data from different observers. The measured as well as estimated $\left(\mathrm{X}_{\mathrm{AA} 1}, \mathrm{Y}_{\mathrm{AA} 1}\right.$, $\left.\mathrm{Z}_{\mathrm{AA} 1}, \alpha, \beta, \gamma\right)$ and their differences of a double-ring marked rod at 4 poses are listed in Table 1 with the angles in degree and distance in $\mathrm{cm}$.

Table I. Experimental Measured/Estimated Parameters (angles in degree, distances in $\mathrm{cm}$ )
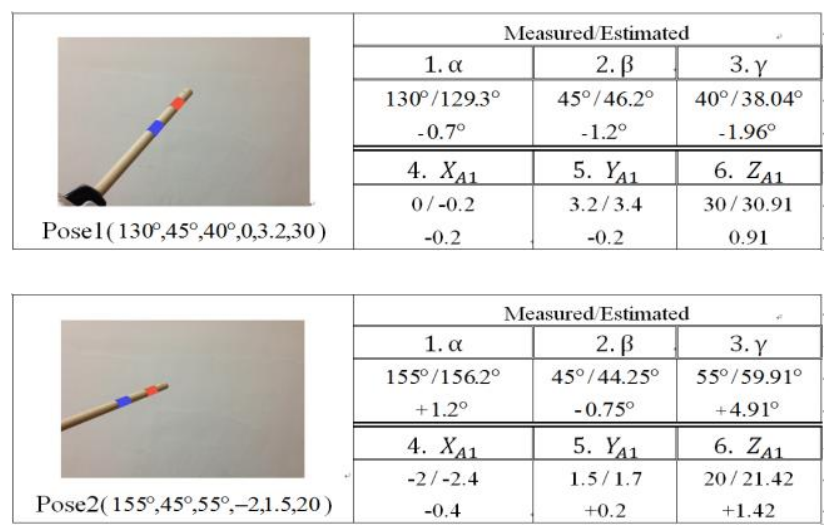

\begin{tabular}{|c|c|c|c|}
\hline \multirow{2}{*}{} & \multicolumn{3}{|c|}{ Measured Estimated } \\
\cline { 2 - 4 } & $1 . \alpha$ & $2 . \beta$ & $3 . \gamma$ \\
\cline { 2 - 4 } & $175^{\circ} / 176.4^{\circ}$ & $10^{\circ} / 10.4^{\circ}$ & $88^{\circ} / 90^{\circ}$ \\
$+1.4^{\circ}$ & $+0.4^{\circ}$ & $+2^{\circ}$ \\
\hline & $4 . X_{A 1}$ & $5 . Y_{A 1}$ & $6 . Z_{A 1}$ \\
\cline { 2 - 4 } Pose $3\left(175^{\circ}, 10^{\circ}, 88^{\circ}, 0,0,28\right)$ & $0 /-0.6$ & $0 / 0.06$ & $28 / 26.9$ \\
\cline { 2 - 4 } & -0.6 & +0.06 & -1.1 \\
\hline
\end{tabular}

\section{Conclusions}

We have proposed an algorithm for estimation of the six $3 \mathrm{D}$ pose parameters for a double-ring marked rod just a single 2D image. Similar to hologram, the 3D pose of a rod shaped object can be reconstructed from a single 2D image or a video frame.

The core of the propsoed algorithm is the equations for estimation of the 6 parameters $\left(\mathrm{X}_{\mathrm{AA} 1}, \mathrm{Y}_{\mathrm{AA} 1}, \mathrm{Z}_{\mathrm{AA} 1}\right.$, $\alpha, \beta, \gamma)$ derived using the camera's perspective transformation relationships between the $3 \mathrm{D}$ rods (with double-ring markers) and the corresponding image. Experimental results prove the correctness of the derived equations and show that the proposed algorithm can estimate the six 3D pose parameters very efficiently with accurately.

It is also noted that the size of the ring markers as well as the distance between the two markers may affect the estimation accuracy. Further improvements on the estimation accuracy as well as estending to rod pose of any orientation is the future research topics.

\section{Acknowledgments}

This paper is supported by Ministry of Science and Technology of Taiwan government under project No. MOST-104-2221-E-224-062 and MOST 103-2221-E-224 -035-MY2 . Also many thanks to Prof. Chuen-Horng Lin of National Taichung University of Science and Technology, 
for his help in derivation of equations, also thank $\mathrm{Mr}$.

Wei-Shih Cheng, Chia-Lun Hsu, students of EE department, NYUST, Yunlin, Taiwan for their help in the experiments.

\section{References}

(1) Zijian Zhao," Real-Time 3D Visual Tracking of Laparoscopic IEEE Instruments for Robotized Endoscope Holder," 11th World Congress on Intelligent Control and Automation (WCICA), Shenyang,pp.6145 pp.6150 June 29 2014-July 42014.

(2) Ching-Chun Huang, Nguyen Manh Hung, and Atul Kumar, "A Hybrid Method for 3D Instrument Reconstruction and Tracking in Laparoscopy Surgery," International Conference on Automation and Information Sciences (ICCAIS)2013, Nha Trang, pp. 36 - 41, 25-28 Nov.2013.

(3) Kai Tai Song and Chun Ju Chen, "Autonomous and stable tracking of endoscope instrument tools with monocular camera," The 2012 IEEE/ASME International Conference on Advanced Intelligent Mechatronics, pp. 39 - 44, July 2012.

(4) G. Q. Wei, K. Arbter, G. Hirzinger, "Real-time visual servoing for laparoscopic surgery," IEEE Engineering in Medicine and Biology, vol. 16, pp 40-45, 1997.

(5) Sandrine Voros, Emmanuel Orvain, Philippe Cinquin, Jean Alexandre Long, "Automatic detection of instruments in laparoscopic images: a first step towards high level command of robotized endoscopic holders," IEEE/RAS-EMBS International Conference on Biomedical Robotics and Biomechatronics, pp. 1107 - 1112, 2006 